Int. Journal of Math. Analysis, Vol. 7, 2013, no. 47, 2333 - 2339

HIKARI Ltd, www.m-hikari.com

http://dx.doi.org/10.12988/ijma.2013.36150

\title{
Problems of Sensor Placement for Intelligent Environments of Robotic Testbeds
}

\author{
Andrey Sheka \\ Department of Intelligent Systems and Robotics \\ Ural Federal University \\ 620083 Ekaterinburg, Russia \\ Andrey.Sheka@gmail.com
}

Copyright (C) 2013 Andrey Sheka. This is an open access article distributed under the Creative Commons Attribution License, which permits unrestricted use, distribution, and reproduction in any medium, provided the original work is properly cited.

\begin{abstract}
In this paper, we consider problems of sensor placement for intelligent environments of robotic testbeds. In particular, we consider the problem of sensor placement and the problem of sensor placement for triangulation based localization. We present experimental results for these problems. Also, we use artificial physics optimization algorithms for solution of the problem of sensor placement. We consider artificial physics optimization for different virtual forces. In particular, we use Runge Kutta neural networks for the calculation of values of virtual forces.
\end{abstract}

Mathematics Subject Classification: 65-05, 68T40, 65L06

Keywords: sensor placement, satisfiability, artificial physics optimization, virtual force, Runge Kutta neural network

\section{Introduction}

Intelligent environments are utilize various sensor networks for many different purposes. In particular, we can mention security-oriented sensor placement 
in intelligent buildings (see e.g. [1]), condition-based placement of distributed active vision sensors for guiding robots in intelligent environment (see e.g. [2], and optimal sensor placement for hot server detection in data centers (see e.g. [3]). In this paper, we consider algorithms for sensor placement in intelligent environments of robotic testbeds.

There are a number of different problems of sensor placement for intelligent environments of robotic testbeds. In particular, it should be mentioned coverage problems for wireless sensor networks, coverage problems for visual surveillance systems, coverage problems for distributed energy supply systems, different problems of placement visual landmarks, etc.

Note that coverage and placement problems for sensors extensively studied (see e.g. [4] - [10]). In particular, there are a number of different algorithms for sensor placement problems (see e.g. [11]). It should be noted that different sensor placement problems received a lot of attention recently (see e.g. [12] [18]). In particular, it were obtained explicit polynomial reductions from the problem of sensor placement SP to MAXSAT, SAT and 3SAT (see $[14,15]$ ). Explicit polynomial reductions from the decision version of the problem of sensor placement for triangulation based localization SPP to SAT and 3SAT were proposed in [18]. It is easy to see that we can use SP and SPP to solve problems of sensor placement for intelligent environments of robotic testbeds.

Note that explicit reductions allow us to obtain exact solutions for sensor placement problems. It is clear that we can use exact solutions as training sets for different intelligent algorithms. In this paper, we consider artificial physics optimization algorithms (see e.g. [19] - [21]) for solution of SP and SPP.

\section{SAT Solvers for SP and SPP}

In this section, we consider SAT solvers for explicit polynomial reductions from SP to MAXSAT and 3SAT (see $[14,15]$ ) and for the explicit polynomial reduction from SPP to 3SAT (see [18]). Note that some SAT solvers for SP and SPP were considered in $[16,18]$. In this section, we consider

A1 genetic algorithm with expansion and exploration operators for MAXSAT (see $[22])$;

A2 GSAT with adaptive score function (see [23]);

A3 genetic algorithm with exons and introns (see [24]);

A4 genetic algorithm with expansion operator (see [25]).

Let $\mathbf{Z}$ be some set of integers. Let $R \subseteq \mathbf{Z}^{2}$ be a discretized workspace for SP. Following [14], we assume that $N \subseteq R$ is a set of target locations. Respectively, $S \subseteq R$ is a set of candidate sensor locations. 
We use heterogeneous cluster (500 calculation nodes, Intel Core i7). We use the generator of natural instances for SP (see [16]). We consider instances with $N$ and $S$ from 400 to 600 (see [16]). Selected experimental results for SP are given in Tables 1,2 .

\begin{tabular}{|l|lll|}
\hline time & average & $\max$ & best \\
\hline fgrasp (see [26]) & $38.1 \mathrm{~min}$ & $11.27 \mathrm{~h}$ & $2.12 \mathrm{~min}$ \\
posit (see [26]) & $42.32 \mathrm{~min}$ & $8.26 \mathrm{~h}$ & $2.97 \mathrm{~min}$ \\
SGA (see [16]) & $1.14 \mathrm{~h}$ & $21.84 \mathrm{~h}$ & $2.17 \mathrm{~min}$ \\
OA (see [16]) & $17.77 \mathrm{~min}$ & $8.18 \mathrm{~h}$ & $19 \mathrm{sec}$ \\
A2 & $19.44 \mathrm{~min}$ & $6.38 \mathrm{~h}$ & $11.2 \mathrm{sec}$ \\
A3 & $4.07 \mathrm{~min}$ & $41.62 \mathrm{~min}$ & $1.1 \mathrm{~min}$ \\
A4 & $6.15 \mathrm{~min}$ & $4.2 \mathrm{~h}$ & $6 \mathrm{sec}$ \\
\hline
\end{tabular}

Table 1: Experimental results for explicit polynomial reduction from SP to $3 \mathrm{SAT}$

\begin{tabular}{|l|lll|}
\hline time & A1 & SGA (see [16]) & OA (see [16]) \\
\hline average & $6.19 \mathrm{~min}$ & $52.1 \mathrm{~min}$ & $32.16 \mathrm{~min}$ \\
max & $12.74 \mathrm{~min}$ & $21.3 \mathrm{~h}$ & $17.6 \mathrm{~h}$ \\
best & $1.5 \mathrm{~min}$ & $53 \mathrm{sec}$ & $11 \mathrm{sec}$ \\
\hline
\end{tabular}

Table 2: Experimental results for explicit polynomial reduction from SP to MAXSAT

Now we consider SAT solvers for SPP. We assume that $W$ is a set of target locations. Respectively, $Q$ is a set of candidate sensor locations. Let $W=\{1,2, \ldots, m\}$ and $Q=\{1,2, \ldots, n\}$. Selected experimental results for SPP are given in Tables $3-5$.

\begin{tabular}{|l|lll|}
\hline time & average & $\max$ & best \\
\hline fgrasp (see [26]) & $23.4 \mathrm{~min}$ & $1.37 \mathrm{~h}$ & $3.19 \mathrm{~min}$ \\
posit (see [26]) & $29.82 \mathrm{~min}$ & $2.09 \mathrm{~h}$ & $2.41 \mathrm{~min}$ \\
GA (see [18]) & $45.36 \mathrm{~min}$ & $3.62 \mathrm{~h}$ & $28 \mathrm{sec}$ \\
A2 & $12.17 \mathrm{~min}$ & $52.44 \mathrm{~min}$ & $8.5 \mathrm{sec}$ \\
A3 & $2.26 \mathrm{~min}$ & $21.7 \mathrm{~min}$ & $37.3 \mathrm{sec}$ \\
A4 & $3.59 \mathrm{~min}$ & $1.18 \mathrm{~h}$ & $5.32 \mathrm{sec}$ \\
\hline
\end{tabular}

Table 3: Experimental results for explicit polynomial reduction from SPP to 3SAT where $n=m=300$ 


\begin{tabular}{|l|lll|}
\hline time & average & $\max$ & best \\
\hline fgrasp (see [26]) & $42.3 \mathrm{~min}$ & $2.92 \mathrm{~h}$ & $8.92 \mathrm{~min}$ \\
posit (see [26]) & $58.4 \mathrm{~min}$ & $4.7 \mathrm{~h}$ & $9.14 \mathrm{~min}$ \\
GA (see [18]) & $1.03 \mathrm{~h}$ & $5.13 \mathrm{~h}$ & $33.4 \mathrm{sec}$ \\
A2 & $26.8 \mathrm{~min}$ & $1.22 \mathrm{~h}$ & $9.16 \mathrm{sec}$ \\
A3 & $3.19 \mathrm{~min}$ & $24.9 \mathrm{~min}$ & $44.1 \mathrm{sec}$ \\
A4 & $9.11 \mathrm{~min}$ & $1.53 \mathrm{~h}$ & $6.22 \mathrm{sec}$ \\
\hline
\end{tabular}

Table 4: Experimental results for explicit polynomial reduction from SPP to $3 \mathrm{SAT}$ where $n=m=350$

\begin{tabular}{|l|lll|}
\hline time & average & $\max$ & best \\
\hline fgrasp (see [26]) & $2.56 \mathrm{~h}$ & $7.11 \mathrm{~h}$ & $22.3 \mathrm{~min}$ \\
posit (see [26]) & $2.43 \mathrm{~h}$ & $9.48 \mathrm{~h}$ & $28.7 \mathrm{~min}$ \\
GA (see [18]) & $2.19 \mathrm{~h}$ & $6.2 \mathrm{~h}$ & $53.55 \mathrm{sec}$ \\
A2 & $39.72 \mathrm{~min}$ & $3.79 \mathrm{~h}$ & $15.88 \mathrm{sec}$ \\
A3 & $5.7 \mathrm{~min}$ & $26.1 \mathrm{~min}$ & $45.9 \mathrm{sec}$ \\
A4 & $14.55 \mathrm{~min}$ & $1.78 \mathrm{~h}$ & $7.41 \mathrm{sec}$ \\
\hline
\end{tabular}

Table 5: Experimental results for explicit polynomial reduction from SPP to $3 \mathrm{SAT}$ where $n=m=600$

\section{An Artificial Physics Optimization for the Problem of Sensor Placement}

In this section, we consider an artificial physics optimization algorithm for SP. In particular, we consider artificial physics optimization for different virtual forces. Also, we use Runge Kutta neural networks (see e.g. [27]) for the calculation of values of virtual forces.

Note that various nature-inspired heuristics have proven very effective for solving different global optimization problems (see e.g. [19]). In particular, the artificial physics optimization is motivated by the success of "Artificial Physics" as a metaphor for controlling multi-robot systems (see [28, 29]). Artificial physics optimization algorithms use three procedures: initialization, calculation force and motion (see [19]). In an artificial physics optimization algorithm, a swarm of individuals is sampled randomly from a problem space in the initialization. Masses of individuals of the swarm should be calculated in the procedure of calculation force. The procedure of motion uses the total force to calculate the velocity of individuals. Note that a felicitous design of force law can drive individuals search problem space intelligently and efficiently. Therefore, the main advantage of artificial physics optimization algorithms consists 
in the proper design of force law.

Different virtual forces are considered (see e.g. [19]). In particular, we can mention negative exponential force law (NEFL), unimodal force law (UFL), linear force law (LFL) [19]. It is well-known that Runge Kutta neural networks can be used for the prediction of different nonlinear systems [27]. Therefore, we use Runge Kutta neural networks for the design of a general force law (RKFL).

We consider 4-order Runge Kutta neural networks with multilayer perceptron networks. We use a gradient learning algorithm for 4-order Runge Kutta neural networks.

Let $T_{\text {opt }}$ be the number of sensors for an optimal solution of SP. Let $T_{A}$ be the number of sensors for a solution of SP with the algorithm $A$. Let

$$
M_{A}=\frac{T_{A}}{T_{o p t}} .
$$

We can consider $M_{A}$ as a measure of the quality of the algorithm $A$. Selected experimental results are given in the Table 6 .

\begin{tabular}{|l|ccc|}
\hline & $5 \cdot 10^{2} \leq N \leq 10^{3}$ & $5 \cdot 10^{3} \leq N \leq 10^{4}$ & $5 \cdot 10^{4} \leq N \leq 10^{5}$ \\
& $10^{2} \leq S \leq 5 \cdot 10^{2}$ & $10^{3} \leq S \leq 5 \cdot 10^{3}$ & $10^{4} \leq S \leq 5 \cdot 10^{4}$ \\
\hline$M_{N E F L}$ & 1.542 & 1.789 & 2.116 \\
$M_{U F L}$ & 1.873 & 2.442 & 3.715 \\
$M_{L F L}$ & 2.292 & 3.788 & 5.204 \\
$M_{R K F L}$ & 1.076 & 1.138 & 1.157 \\
\hline
\end{tabular}

Table 6: An average number of sensors for different algorithms.

\section{References}

[1] D. G. Eliadesa, M. P. Michaelidesa, C. G. Panayiotoua, and M. M. Polycarpou, Security-oriented sensor placement in intelligent buildings, Building and Environment, 63 (2013), 114-121.

[2] J.-H. Lee, K. Morioka, N. Ando, and H. Hashimoto, Condition-based placement of distributed active vision sensors for guiding robots in intelligent environment, 2003 IEEE/ASME International Conference on Advanced Intelligent Mechatronics, 1 (2003), 546-551.

[3] X. Wang, X. Wang, G. Xing, J. Chen, C.-X. Lin, and Y. Chen, Towards Optimal Sensor Placement for Hot Server Detection in Data Centers, Proceedings of the 2011 31st International Conference on Distributed Computing Systems, (2011), 899-908. 
[4] S. Aranda, S. Martinez, and F. Bullo, On optimal sensor placement and motion coordination for target tracking, Proceedings of the 2005 IEEE International Conference on Robotics and Automation, (2005), 4544-4549.

[5] A. Efrat, J. S. B. Mitchell, and S. Har-Peled, Approximation algorithms for two optimal location problems in sensor networks, 2nd International Conference on Broadband Networks, 1 (2005), 714-723.

[6] V. Isler, S. Khanna, J. Spletzer, and C. Taylor, Target tracking with distributed sensors: The focus of attention problem, Computer Vision and Image Understanding, 100 (2005), 225-247.

[7] S. Martinez and F. Bullo, Optimal sensor placement and motion coordination for target tracking, Automatica, 42 (2006), 661-668.

[8] J. Spletzer and C. Taylor, Dynamic sensor planning and control for optimally tracking targets, International Journal of Robotics Research, 22 (2003), 7-20.

[9] S. Susca, S. Martinez, and F. Bullo, Monitoring Environmental Boundaries with a Robotic Sensor Network, IEEE Transactions on Control Systems Technology, 16 (2008), 288-296.

[10] O. Tekdas and V. Isler, Sensor Placement for Triangulation Based Localization, IEEE Transactions on Automation Science and Engineering, 7 (2010), 681-685.

[11] A. Osmani, Design and Evaluation of New Intelligent Sensor Placement Algorithm to Improve Coverage Problem in Wireless Sensor Networks, Journal of Basic and Applied Scientific Research, 2 (2012), 1431-1440.

[12] V. Popov, Partially Distinguishable Guards, Applied Mathematical Sciences, 6 (2012), 6587-6591.

[13] A. Gorbenko and V. Popov, The Problem of Selection of a Set of Partially Distinguishable Guards, Applied Mathematical Sciences, 7 (2013), 651654 .

[14] A. Gorbenko, M. Mornev, V. Popov, and A. Sheka, The Problem of Sensor Placement, Advanced Studies in Theoretical Physics, 6 (2012), 965-967.

[15] A. Gorbenko and V. Popov, On the Problem of Sensor Placement, Advanced Studies in Theoretical Physics, 6 (2012), 1117-1120.

[16] A. Gorbenko and V. Popov, SAT Solvers for the Problem of Sensor Placement, Advanced Studies in Theoretical Physics, 6 (2012), 1235-1238. 
[17] A. Gorbenko and V. Popov, Clustering Algorithm in Mobile Ad Hoc Networks, Advanced Studies in Theoretical Physics, 6 (2012), 1239-1242.

[18] A. Gorbenko, M. Mornev, V. Popov, and A. Sheka, The problem of sensor placement for triangulation-based localisation, International Journal of Automation and Control, 5 (2011), 245-253.

[19] L. Xie and J. Zeng, The Performance Analysis of Artificial Physics Optimization Algorithm Driven by Different Virtual Forces, ICIC Express Letters, 4 (2010), 239-244.

[20] A. Gorbenko and V. Popov, The Force Law Design of Artificial Physics Optimization for Robot Anticipation of Motion, Advanced Studies in Theoretical Physics, 6 (2012), 625-628.

[21] A. Gorbenko and V. Popov, The Force Law Design of Artificial Physics Optimization for Starting Population Selection for GSAT, Advanced Studies in Theoretical Physics, 7 (2013), 131-134.

[22] A. Gorbenko and V. Popov, A Genetic Algorithm with Expansion and Exploration Operators for the Maximum Satisfiability Problem, Applied Mathematical Sciences, 7 (2013), 1183-1190.

[23] V. Popov, GSAT with Adaptive Score Function, Advanced Studies in Theoretical Physics, 7 (2013), 363-366.

[24] V. Popov, Genetic Algorithms with Exons and Introns for the Satisfiability Problem, Advanced Studies in Theoretical Physics, 7 (2013), 355-358.

[25] V. Popov, A Genetic Algorithm with Expansion Operator for the 3Satisfiability Problem, Advanced Studies in Theoretical Physics, 7 (2013), 359-361.

[26] URL: http://people.cs.ubc.ca/ hoos/SATLIB/

[27] Y.-J. Wang and C.-T. Lin, Runge Kutta neural network for identification of continuous systems, Proceedings of the 1998 IEEE International Conference on Systems, Man, and Cybernetics, (1998), 3277-3282.

[28] W. M. Spears, D. F. Spears, J. Hamann, and R. Heil, Distributed, PhysicsBased Control of Swarms of Vehicles, Autonomous Robots, 17 (2004), 137162.

[29] W. M. Spears, D. F. Spears, R. Heil, W. Kerr, and S. Hettiarachchi, An Overview of Physicomimetics, Lecture Notes in Computer Science, 3342 (2005), 84-97.

\section{Received: June 15, 2013}

